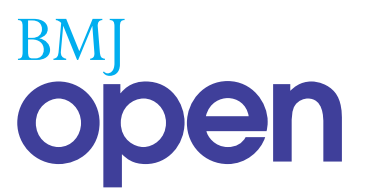

\title{
Patients' subjective concepts about primary healthcare utilisation: the study protocol of a qualitative comparative study between Norway and Germany
}

\author{
Wolfram J Herrmann, ${ }^{1}$ Alexander Haarmann, ${ }^{1}$ Uwe Flick, ${ }^{2}$ Anders Bærheim, ${ }^{3}$ \\ Thomas Lichte, ${ }^{1}$ Markus Herrmann ${ }^{1}$
}

To cite: Herrmann WJ, Haarmann A, Flick U, et al. Patients' subjective concepts about primary healthcare utilisation: the study protocol of a qualitative comparative study between Norway and Germany. BMJ Open 2013;3: e002952. doi:10.1136/ bmjopen-2013-002952

\section{- Prepublication history for this paper is available online. To view these files please visit the journal online (http://dx.doi.org/10.1136/ bmjopen-2013-002952).}

Received 26 March 2013 Accepted 10 April 2013

This final article is available for use under the terms of the Creative Commons Attribution Non-Commercial 2.0 Licence; see http://bmjopen.bmj.com

${ }^{1}$ Institute of General Practice and Family Medicine, Ottovon-Guericke-University Magdeburg, Magdeburg, Germany

${ }^{2}$ Alice Salomon Hochschule University of Applied Sciences, Berlin, Germany ${ }^{3}$ Department of Global Public Health and Primary Care, University of Bergen, Bergen, Norway

Correspondence to Dr Wolfram J Herrmann; wolfram.herrmann@med. ovgu.de

\section{ABSTRACT}

Background: In Germany, utilisation of ambulatory healthcare services is high compared with other countries: While a study based on the process data of German statutory health insurances showed an average of 17.1 physician-patient-contacts per year, the comparable figure for Norway is about five. The usual models of healthcare utilisation, such as Rosenstock's Health Belief Model and Andersen's Behavioural Model, cannot explain these differences adequately.

Organisational factors of the healthcare system, such as gatekeeping, do not explain the magnitude of the differences. Our hypothesis is that patients' subjective concepts about primary healthcare utilisation play a major role in explaining different healthcare utilisation behaviour in different countries. Hence, the aim of this study is to explore these subjective concepts comparatively, between Germany and Norway.

Methods/design: With that aim in mind, we chose a comparative qualitative study design. In Norway and Germany, we are going to interview 20 patients each with qualitative episodic interviews. In addition, we are going to conduct participant observation in four German and four Norwegian primary care practices. The data will be analysed by thematic coding. Using selected categories, we are going to conduct comparative case and group analyses.

Ethics and dissemination: The study adheres to the Declaration of Helsinki. All interviewees will sign informed consent forms and all patients will be observed during consultation. Strict rules for data security will apply. Developed theory and policy implications are going to be disseminated by a workshop, presentations for experts and laypersons and publications.

\section{BACKGROUND}

The utilisation of ambulatory healthcare is comparatively high in Germany. The beneficiaries of statutory health insurances had an average of 17.1 contacts with physicians per person per year in 2007 in ambulatory care. ${ }^{1}$
This figure is considerably higher compared with those in other countries. The number of contacts for Norway, for example, amounts to 4.6 per person per year, ${ }^{2}$ a country which is comparable with Germany regarding mortality and morbidity among the population. The reasons for this significantly higher utilisation of ambulatory care in Germany compared with other countries such as Norway are largely unexplored. In this research project, utilisation of primary healthcare is understood as the number of contacts of a patient with a general practitioner (GP) per time unit.

Way back in 1966, Rosenstock posed the question 'Why People Use Health Services'. He developed his so-called Health Belief Model on the utilisation of preventive and diagnostic healthcare provision. The Health Belief Model comprises two classes of variables: These are the readiness to get active and assumptions about the effectiveness of different measures.

The 'Behavioural model on the utilization of healthcare provision' published by Andersen for the first time in 1968 is also based on the idea that high-utilisation rates in healthcare are a positive phenomenon and will lead to better healthcare outcomes. ${ }^{4}$ Andersen distinguished predisposing factors such as demographic factors, social structure and health beliefs, enabling factors such as family, community and the perceived need for healthcare. Later on, Andersen extended his model with characteristics of the healthcare system (policy, resources, organisation) and fitted a feedback loop into his model. In Andersen's model, health beliefs are defined as attitudes, values and knowledge about health and healthcare provision. However, quantitative studies which apply Andersen's model rarely make use of the factor 'health 
beliefs', which can mainly be attributed to difficulties in operationalisation. When it comes to the effect of attitudes, values and knowledge about healthcare provision between different countries within the framework of Andersen's model, we do not know of any such study to the best of our knowledge.

On the basis of the models by Rosenstock and Andersen, numerous studies were conducted in order to get a better understanding of the predictors about individual utilisation behaviour. Exemplary studies focusing on the German healthcare model are the studies by Thode $e t a l,{ }^{5}$ Bergmann et $a l^{6}$ and Kürschner et al. ${ }^{7}$ The results of these studies demonstrate the importance of age, morbidity and sex to the extent of utilisation of healthcare. Since there are no major dissimilarities regarding the factors age, morbidity and sex in the overall population in western countries such as Germany and Norway, these factors are unable to explain the vast differences in healthcare utilisation.

Another starting point for the explanation of utilisation behaviour relates to factors inherent to the healthcare system, specifically whether the GP functions as a gatekeeper to specialists. Garrido et $a l^{8}$ examined in a systematic review the effect of gatekeeping on the utilisation of physicians working in ambulatory care. A tendency towards a reduced utilisation of specialists could be demonstrated, which to some extent was compensated by a higher recourse to GP. Hence, the effect of gatekeeping is not able to explain the magnitude of the differences found in utilisation of ambulatory care either.

Thus, we focus on health beliefs as defined in Andersen's model, ${ }^{4}$ which have been widely neglected by scholars so far, as an important factor for different healthcare utilisation in different countries. A broader concept regarding health beliefs is subjective concepts. We view subjective concepts as underlying assumptions, values and knowledge with regard to a specific object. Hence, we define subjective concepts of the utilisation of ambulatory healthcare as the assumptions of patients, how and why they decide to make use of ambulatory healthcare given a specific health issue, as well as the underlying attitudes, values and knowledge leading to this decision. In turn, we see these subjective concepts as influenced by society. This definition of subjective concepts is guided by subjective layman theories, which contain an implicit structure of reasoning as described by Groeben et al. ${ }^{9}{ }^{10}$ The second base is the 'Theory of social representation', which describes the societal contingency of subjective concepts. ${ }^{11}$

As individual concepts of patients are (also) influenced by society, differing subjective concepts can be expected in different societies. Subjective concepts can therefore potentially explain differences in utilisation in several countries. Figure 1 depicts the theoretical framework of the study.

As a country of comparison for the concepts of German patients, Norway is well suited, because of its state-run healthcare system contrasting with Germany's

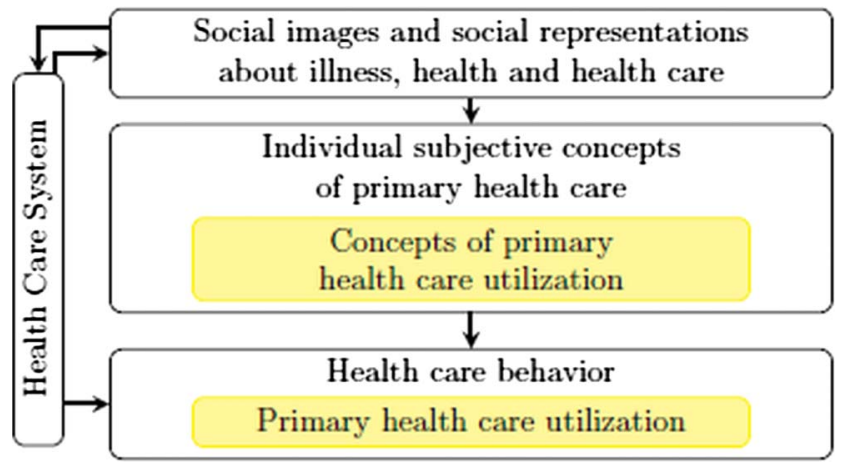

Figure 1 Theoretical framework of the research project.

cooperative one with mainly self-employed GP in both systems. $^{12}$ The cultural differences between Germany and Norway are small in scale, allowing for a comparison. Life expectancy in Norway can be compared with the one in Germany, whereas the utilisation of ambulatory care differs widely. ${ }^{2}$ With regard to experiences of physicians who have worked in both systems, ${ }^{2}{ }^{13}$ as well as differences in medical guidelines, ${ }^{14}$ it can be presumed that Norwegian and German patients hold different views on the utilisation of ambulatory care.

\section{AIMS}

Our aim is to research the patients' subjective concepts which determine the different healthcare utilisation behaviour in Germany and Norway. Thus, our research question is:

How do adult patients' subjective concepts about primary healthcare utilization differ between Germany and Norway?

\section{METHODS}

Our methodological starting point is the patient's own experiences and frequent reasons for encounter. The research design is qualitative: patients in Germany and Norway are going to be interviewed. In addition, we are going to conduct participant observation in primary healthcare practices. The data from both methodological approaches are going to be triangulated. We are going to compare cases and groups based on thematic coding.

\section{Methodological approach}

In order to conduct research on cultural differences, several methodological approaches are available: for example, participant observation is widely common in ethnography, ${ }^{15}$ and the episodic interview is used to compare groups by thematic coding. ${ }^{16}$ The underlying theoretical assumption in both approaches is that individual perceptions and concepts are at least partially socially constructed. 
Figure 2 Methodological approach and the theoretical framework of the research project.

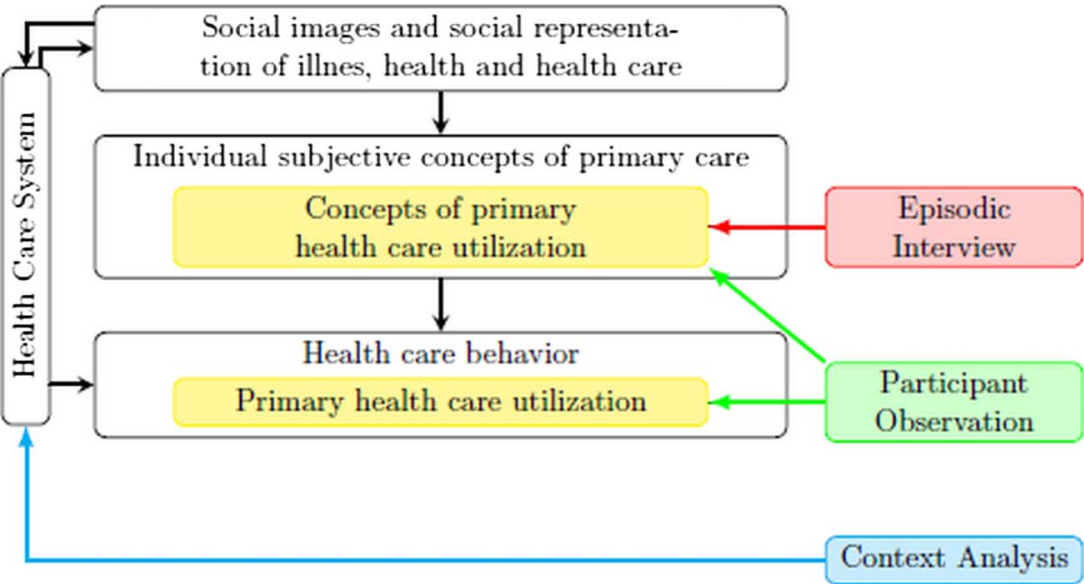

In this project, both methodological approaches shall be applied and triangulated. ${ }^{17}$ Figure 2 gives an overview of the project's theoretical framework, completed by the respective data collection instruments: both episodic interview and participant observation apply different methodological approaches to research the subjective concepts of patients and the resulting behaviour. The usage of both methods and their triangulation shall lead to broad, rich and valid results.

\section{Data collection methods}

In many studies, qualitative interviews have been employed to research subjective concepts, since qualitative interviews provide access to individual cognitions and their world of experiences. In this context, Flick ${ }^{16-18}$ has developed the method of the 'episodic interview' specifically designed to compare the subjective concepts of specific groups. In episodic interviews, questions posed in order to evoke narrations are combined with questions aiming at argumentative-theoretical answers.

The topics and the narrative stimuli will be covered by an interview guideline. The guideline does not imply a certain order of the respective questions, but rather serves as an aid to memory. The aim is to get into a conversation with the interviewee, which resembles a conversation, under which course the respective parts on the guideline will be touched upon. In this study, the guideline has already been developed and tested (cf. box 1).

Participant observation as a method relies on being present in the field, that is, in the setting, among the individuals and surroundings to be researched. Next to the actors' perceptions in the field, the action based on them is on the focus of this particular method. Thus, we intend to observe how patients utilising primary healthcare express their reasons for utilisation and assumptions of care in the GP's practice. This can, for example, be at the reception desk, in the waiting area, during the consultation with the GP, in the interaction with other patients, the staff or the GP. The projected time in each
GP's practice is 1 week. The researcher attempts to get into a conversation with the actors involved, in order to explore their underlying point of view for their actions. A regular retreat of the researcher in order to note down the observations is a characteristic of the method and secures the quality of the data. In order to improve the quality and comparability of the participant observation, an observation guideline will be used, which lists the general framework and objectives for the observation as well as the notes. This guideline has already been developed (cf. box 2).

\section{Sampling}

Concerning the participant observation, we base our sample on a $2 \times 2$ design: Of the overall eight observations, lasting for 1 week each, half will be conducted in Germany, and the other half in Norway. In order to account for the potential differences in the availability of services and the socioeconomic standing of different types of municipalities, it will be differentiated between a rather urban setting and a more rural area in each country (cf. Refs. ${ }^{19-21}$ ). That yields the distribution of participant observations as summarised in figure 3 . The 81-week participant observations facilitate a very broad design accounting for differences, while, at the same time, keeping the volume of qualitative data material within manageable limits.

The number of interviewees also aims at reaching a relevant amount of comparison with a contentwise saturation, which at the same time still allows for a deeper analysis of the collected data. ${ }^{22}$ Therefore, 40 patients shall be interviewed, half of them in Germany, and the other half in Norway. Figure 3 provides an overview of the sampling procedure.

Contact with potential participants in the study shall be established through the eight cooperating GPs' practices. All patients visiting on any of two certain days will consecutively receive an information sheet asking for the declaration of consent to be contacted by the research team and a short survey on age, sex, subjective 


\section{Box 1 Interview guideline}

This interview guideline is not to be followed strictly, but a guide as to what questions could be asked and which topics should be covered during the interview. The interview itself should tend to be a dialogue.

Preamble: Explain the interview and the purpose. We have as much time as you would like to spend.

When did you visit a physician the last time? Can you tell me the situation? (if the last physician was a general practitioner (GP)/ not a GP, ask vice versa again).

Why do you mostly go to the physician?-Can you tell me more about the last time this happened?

When did you recently think about going to the physician, but did not do it?-Can you tell me about this situation?

When do you not go to a physician, but others might?

When do you go to a physician, but others might not?

What do you expect from your physician?

From your opinion, which are the problems a GP is responsible for? For which problems is he not?

When was the last time you did not go to a GP but to another specialist?-Can you tell me this situation?

When do you prefer not to go to a GP but to another specialist?

Have you ever directly used Legevakt/Kassenärztlichen Bereitschaftsdienst? Can you please tell me more about this situation?

Have you ever gone to a physician because you needed a sickness certification? Can you please tell me more about this situation?

Can you remember the last time you suffered from low back pain? Can you please tell me about the situation and what you did?

Can you remember the last time you suffered from a cough/cold? Can you please tell me about the situation and what you did?

Can you remember the last time you suffered from diarrhoea? Can you please tell me about the situation and what you did?

Have you ever had a tick bite? (if not, go for mosquito bite) Can you please tell me about the situation, the last time you had a tick bite? Have you ever thought of visiting your GP because of a tick bite?

If you have a medical problem for which the reason is unclear, what do you expect from your GP?

The costs you have from visiting a GP, has this ever influenced your decision to visit a GP?-Can you tell me this situation?

How long do you need to drive to a GP? Has this ever influenced your decision to visit a GP? -Can you tell me this situation?

How long do you have to wait to get an appointment with your GP? Has this ever influenced your decision to visit a GP?-Can you tell me this situation?

What do you think is the reason for the difference in healthcare utilisation between Germany and Norway?

What do you think is important and that we have not mentioned in the interview yet?

morbidity and the number of contacts with a physician during the last 3 months. Out of this range of potential interviewees, the actual participants will be picked in an iterative process. Therefore, interviewees will be chosen evenly from both sexes and different age groups. In order to arrive at comparable results, German interviewees shall be matched with the Norwegian ones with regard to age, sex, subjective morbidity and healthcare

\section{Box 2 Observation guideline}

\section{Place of observation \\ - Reception \\ Waiting room \\ - Consultation room \\ Focus of observation \\ - Reason for encounter \\ - Communication of this reason \\ - Expectations mentioned \\ - Communication of wishes, demands and expectations \\ - Referrals, new appointments, results of consultation \\ - Approach and treatment of patients by physician and staff \\ - On a sideline: social chit-chat}

utilisation. The German interviewees will be chosen according to the Norwegian participants. For matching a point, the system evaluates the match with a score of 5 points as best matching and 4 or 4.5 points as good matching. Table 1 displays the matching criteria. The quartiles concerning subjective chronic diseases and visits to a physician are calculated separately in each country for urban and rural areas.

After conducting half of the interviews and a first rough analysis, it will be decided whether or not patients generally not visiting any GP or specialist are sufficiently represented in the sample. It might be necessary to access them via a different sampling procedure in the second round of interviews.

\section{Data analysis}

The analysis of the qualitative data material is based on the concept of Grounded Theory. ${ }^{23}$ The basic underlying idea of Grounded Theory is to develop a specific, short-range or middle-range 'theory' in a stepwise process of analysis, that is, 'grounded' in the data. Theoretical coding is at the heart of the analytical process, for which codes are assigned to certain text segments. What is different from many other coding schemes is that the codes are not set beforehand but emerge during the process. Data collection and analysis occur step by step, not sequentially, but parallel with each other.

Based on theoretical coding, thematic coding was developed by Flick ${ }^{24}$. An essential dissimilarity between the two of them is that the latter first takes individual cases into account and, second, facilitates comparisons between groups. While theoretical coding dissolves the single cases into the overall data material, the relation to the individual case is preserved in thematic coding. The reference to the case is ensured through a description of each individual case. In addition, it facilitates the development of typologies for specific aspects of the researched topic based on the respective cases. The project will make use of the aforementioned possibility of comparison of groups, where interviewees in Norway 
Figure 3 Sampling of Interviewees.

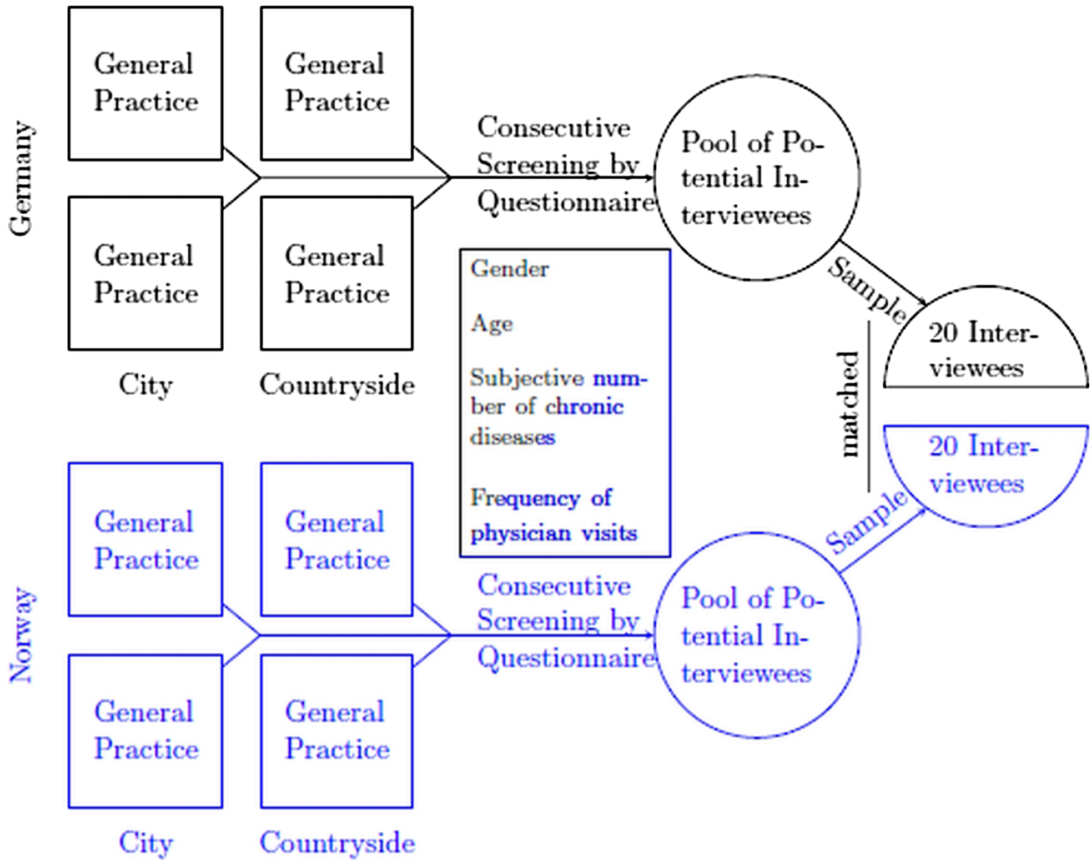

and interviewees in Germany are going to comprise the two main groups.

The interviews will be transcribed word for word, after which a rough case description will be developed for each case. To begin with, when it comes to the analysis, two interviews from Norway and the same number from Germany will be chosen according to the method of maximising the contrast between the respective cases. ${ }^{25}$ The chosen interviews will be coded line by line ${ }^{26}$ that is, an ad hoc code will be assigned to each line. The codes themselves are not predefined. Based on these line by line codes of the four interview transcripts, a thematic structure will be developed. A thematic structure is a system of categories, in which each code can be associated with a category. As a last step, all interviews will be coded subsequently within this thematic structure, which however will be adapted over the course of coding so that new categories can be added or sorted differently. Parallel to coding, the case description will be developed further and refined. The same applies to sequences of the notes taken during the observation.

Line-by-line coding is performed by the first and the second authors. They compare their line-by-line codes

\begin{tabular}{lll} 
Table 1 & Matching criteria & \\
\hline Variable & Criteria & Points \\
\hline Sex & Same & 1 \\
Age difference & $\leq 5$ & 1 \\
& $\leq 10$ & 0.5 \\
Subjective Morbidity & Same quartile & 1 \\
& Adjacent quartile & 0.5 \\
Physician contacts & Same quartile & 1 \\
& Adjacent quartile & 0.5 \\
\hline
\end{tabular}

and discuss the differences and similarities. Coding is performed by the first and the second authors. The coding is reviewed in sessions by the project team. During the process of data analysis, we are going to write memos on the cultural and professional differences emerging. These memos and protocols of sessions of data analysis are collected and seen as part of the interpretation process and quality management.

Based on the thematic categories and the assigned codes and text segments associated with them, a thorough analysis of selected topics can be conducted. With the aid of comparisons between cases and groups, subjective concepts of patients in Norway and Germany can be compared and typologies developed.

\section{Ethical considerations}

The study adheres to the Declaration of Helsinki. All interviewees sign an informed consent as well as all the patients observed during consultation. Strict rules for data security apply: Addresses and contact information are stored physically separate from content data. Any contact information is destroyed as early as possible, for example, after the interview; only anonymised data are used for analysis. The study has been approved by the local ethics committees in Magdeburg/Germany and Bergen/Norway.

\section{Dissemination}

Developed theory and policy implications are going to be disseminated through a workshop for researchers and policymakers. The results are going to be presented at several congresses and conferences, and we are going to publish the results in peer-reviewed journals. In addition, we are going to present our results to laypersons by public talks. 


\section{DISCUSSION}

Conducting a comparative qualitative study creates several difficulties which have to be addressed when planning such a study. An important problem in comparative studies is language. Qualitative interviews, participant observation and data analysis are highly language dependent. Thus, native speakers taking part in the study and having good knowledge of both languages of the core personnel are important for conducting a valid study. An important question is when in the process of analysis to translate the interviews. We decided to code the interviews in the original language and to translate the sections needed for publication in the last step. In our research group, three researchers and one student assistant are able to understand at least written German and at least written Norwegian. This seems fairly convenient to avoid language difficulties; however, it partly excludes those researchers who are not able to read in both languages.

Another difficulty is the funding of a comparative study. National grant agencies usually finance personnel in their own country only. EU funds are usually announced for specified topics and only for projects involving more than two countries. The organisation of data privacy and ethical reviews are different between different countries: for example, in Norway, the emphasis is on the privacy of the physician-patient encounter, while the emphasis in Germany is on data processing and use of personal data. Oeye et $a l^{27}$ have already described the difficulties in obtaining ethical approval for participant observation in the medical field in Norway.

\section{CONCLUSION}

In health services research, it is important not only to state the quantitative differences between countries, but also to look for the reasons for these differences. Qualitative methods are available to compare the subjective and social factors potentially involved in these differences or to explore the possible reasons for the differences. However, comparative qualitative methods are challenging because of the methodological and organisational difficulties occurring.

Acknowledgements The authors would like to thank Michael Freitag, Jochen Gensichen, Thorsten Meyer and Katrin Balzer for constructive comments on earlier versions of this study protocol and helpful ideas. WJH thanks DFG-Nachwuchsakademie Versorgungsforschung, especially Martin Scherer, for making it possible to develop this research idea.

Contributors WJH designed the study and drafted the manuscript. AH helped in drafting and revising the manuscript. $A B$ gave advice on the Norwegian background of the study, helped in designing the study and revised the manuscript. UF gave methodological advice in designing the study and revised the manuscript. TL and MH gave advice on the German background of the study and revised the manuscript. All authors have read and approved the final manuscript. All authors have read and approved the final manuscript.

Funding DFG (German Research Foundation) Reference number HE 6399/1. DFG has no influence on the interpretation of the findings or publication of the results.
Competing interests WJH is employed in a general practice. TL and MH own and run their own general practices. The other authors state having no potentially competing interests.

Ethics approval Local ethics committee at the Medical Faculty, University of Magdeburg, Germany; and REK Vest in Bergen, Norway.

Provenance and peer review Not commissioned; internally peer reviewed.

Data sharing statement No additional data are available.

\section{REFERENCES}

1. Riens B, Erhart M, Mangiapane S. Arztkontakte im Jahr 2007Hintergründe und Analysen. Zentralinstitut für die kassenärztliche Versorgung in Deutschland 2012. http://www.versorgungsatlas.de/ fileadmin/ziva_docs/14/Arztkontakte_Bericht_Langversion.pdf (last checked: 03.06.2013).

2. Karsch-Völk M, Kamps H, Schneider A. Vergleich des deutschen mit dem norwegischen primärarztsystem aus ärztlicher Sicht. Z Allgemeinmed 2010;86:468-73.

3. Rosenstock IM. Why people use health services. Milbank Mem Fund Q 1966;44:94-127.

4. Andersen RM. Revisiting the behavioral model and access to medical care: does it matter? J Health Soc Behav 1995;36:1-10.

5. Thode N, Bergmann E, Kamtsiuris P, et al. Einflussfaktoren auf die ambulante Inanspruchnahme in Deutschland. Bundesgesundheitsblatt Gesundheitsforschung Gesundheitsschutz 2005;48:296-306.

6. Bergmann E, Kalcklösch M, Tiemann F. Inanspruchnahme des Gesundheitswesens. Erste Ergebnisse des telefonischen Gesundheitssurveys 2003. Bundesgesundheitsblatt Gesundheitsforschung Gesundheitsschutz 2005;48:1365-73.

7. Kürschner N, Weidmann C, Müters S. Wer wählt den Hausarzt zum Gatekeeper? Eine Anwendung des Verhaltensmodells von Andersen zur Beschreibung der Teilnahme an Hausarztmodelle in Deutschland. Bundesgesundheitsblatt Gesundheitsforschung Gesundheitsschutz 2011;54:221-7.

8. Garrido MV, Zentner A, Busse R. The effects of gatekeeping: a systematic review of the literature. Scand J Prim Health Care 2011;29:28-38.

9. Groeben N, Scheele B. Argumente für eine Psychologie des reflexiven Subjekts: Paradigmawechsel vom behavioralen zum epistemologischen Menschenbild. Darmstadt: Steinkopff, 1977.

10. Groeben N, Wahl D, Schlee J, et al. Das Forschungsprogramm Subjektive Theorien. Eine Einführung in die Psychologie des reflexiven Subjekts. Tübingen: Francke, 1988.

11. Flick U, ed. Psychologie des Sozialen. Repräsentationen in Wissen und Sprache. Reinbek: Rowohlt, 1995.

12. Johnsen JR. Health systems in Transition: Norway. Vol. 8. Copenhagen: WHO Regional Office for Europe on behalf of the European Observatory on Health Systems and Policies, 2006.

13. Kamps H. Deutsches Gesundheitssystem: Mehr Mut zum NEIN-Sagen. Dtsch Arzteb/ 2007;104:A105-8.

14. Christiaens T, Backer DD, Burgers J, et al. Guidelines, evidence, and cultural factors. Scand J Prim Health Care 2004;22:141-5.

15. Hammersley M, Atkinson P. Ethnography. Principles in practice. London: Routledge, 1995.

16. Flick U. Psychologie des technisierten Alltags. Soziale Konstruktion und Repräsentation technischen Wandels in verschiedenen kulturellen Kontexten. Opladen: Westdeutscher Verlag, 1996.

17. Flick U. Triangulation. Eine Einführung. Wiesbaden: VS Verlag für Sozialwissenschaften, 2008.

18. Flick U. Managing quality in qualitative research. London: SAGE, 2007.

19. Busato A, Matter $P$, Künzi B, et al. Geographic variation in the cost of ambulatory care in Switzerland. J Health Serv Res Policy 2011;17:18-23.

20. Weber I. Alte und neue Bundesländer-Mehr Patienten, weniger Ärzte und geringere Finanzmittel-Unzureichende Ressourcen für die ambulante Versorgung in den neuen Ländern. Dtsch Arztebl 2005;102:A2987-88.

21. Berth H, Wagner W, Brähler E. Kulturschock Deutschland. Eine empirische Untersuchung zu alltagskulturellen Differenzen von Ostund Westdeutschen. Psychosozial 2000;80:9-21.

22. Merkens H. Auswahlverfahren, Sampling, Fallkonstruktion. In: Flick U, Kardorff Ev, Steinke I, eds. Qualitative Forschung. Ein Handbuch. Reinbek: Rowohlt, 2004:286-99.

23. Glaser BG, Strauss AL. Grounded theory. strategien qualitativer forschung. Bern: Hans Huber, 1998. 
24. Flick U. An introduction to qualitative research. London: SAGE, 2009.

25. Kelle U, Kluge S. Vom Einzelfall zum Typus. Fallvergleich und Fallkonstrastierung in der qualitativen Sozialforschung. VS Verlag für Sozialwissenschaften, 2010.
26. Charmaz K. Constructing grounded theory. A practical guide through qualitative analysis. London: SAGE, 2006.

27. Oeye C, Bjelland AK, Skorpen A. Doing participant observation in a psychiatric hospital-research ethics resumed. Soc Sci Med 2007;65:2296-306. 